\title{
Erratum to: Cosmic Ray Spectrum Approximation Model: Experimental Results and Comparison with Other Models
}

\author{
M. Buchvarova $\cdot$ D. Draganov
}

Published online: 26 February 2013

(C) Springer Science+Business Media Dordrecht 2013

\section{Erratum to: Solar Phys \\ DOI 10.1007/s11207-012-0157-8}

We have found four serious and some minor typographical errors in our paper "CosmicRay Spectrum Approximation Model: Experimental Results and Comparison with Other Models".

The first serious error is related to the rigidity loss $\phi$ and the force-field parameter $\varphi$. In particular, the symbol $\phi$ is replaced with $\varphi$ in some places of the article. Additionally, there is a semantic error in Section 3, paragraph 3. The error corrections are given below:

Figure 2: " $\varphi$ " should be changed to " $\phi$ " in the caption of Figure 2, i.e. the correct sentence is: "Local interstellar spectrum $f_{\text {LIS }}(P)$ and modulated cosmic-ray spectrum $f(P)$, described by the distribution function $f ; P^{\prime \prime}-P=\phi, \phi$ is the force-field rigidity loss."

Section 3, paragraph 2: the expression "see Figure 2, where the force-field rigidity loss $\varphi$ is described by a horizontal line" should be replaced by: "see Figure 2, where the force-field rigidity loss $\phi$ is described by a horizontal line".

Section 3, paragraph 3: the expression "the force-field rigidity loss $\varphi$ represented by the horizontal line in Figure 2" should be replaced by: "the force-field rigidity loss $\phi$ represented by the horizontal line in Figure 2".

In Section 3, paragraph 3 the sentence "The relation between $\alpha_{P}, \beta_{P}$ and the modulation potential $\varphi$, given in Section 4.1.2. of Moraal (2011), indicates that $\phi$ is numerically equal to the modulation potential $\varphi$ only in the special case of relativistic particles $(\bar{v} \approx 1)$ and rigidity dependence $k_{2} \sim P$." should be replaced by: "The relation between $\alpha_{P}, \beta_{P}$ and the modulation potential $\varphi$ is given in Section 4.1.2. Moraal (2011) indicates that $\phi$ is numer-

The online version of the original article can be found under doi:10.1007/s11207-012-0157-8.

M. Buchvarova $(\varangle) \cdot$ D. Draganov

Space Research and Technology Institute - Bulgarian Academy of Sciences, Acad. G. Bonchev Str,

Bl.1, Sofia 1113, Bulgaria

e-mail: marusjab@yahoo.com 
ically equal to the force-field parameter $\varphi$ only in the special case of relativistic particles $(\bar{v} \approx 1)$ and for rigidity dependence $k_{2} \sim P . "$

Section 3, paragraph 3: the sentence "In all other cases, the rigidity loss $\varphi$ in Figure 2 cannot be represented by a modulation potential, and the modulation in the force-field formalism is better described using the dimensionless parameter $M$ (Moraal, 2011)." should be replaced by: "In all other cases, the rigidity loss $\phi$ in Figure 2 cannot be represented by a force-field parameter, and the modulation in the force-field formalism is better described using the dimensionless parameter M (Moraal, 2011)."

Section 4.1.2, paragraph 1: the expression "If the force-field rigidity loss $\varphi$ is numerically equal to the modulation potential $\varphi$ and $\beta_{P}^{*} \equiv \tan \theta^{*}$, then the tangent in Figure 2 is" should be replaced by: "If the rigidity loss $\phi$ is numerically equal to the force-field parameter $\varphi$ and $\beta_{P}^{*} \equiv \tan \theta^{*}$, then the tangent in Figure 2 is".

Also, there is a typographical error in formula (21). The correct equation is:

$$
\beta_{P}=\frac{\ln \left(f_{\mathrm{LIS}}(P)\right)-\ln (f(P))}{\ln \left(P+\alpha_{P}\right)-\ln (P)} .
$$

In Section 6, paragraph 1 the sentence "Drift mechanisms have time lags of several months and are characterized by a hysteresis phenomenon (Kane, 2003)." should be replaced by: "Long-term variation in cosmic ray intensity modulation, determined by convectiondiffusion and drift mechanisms, has a time lag of several months and is characterized by a hysteresis phenomenon (Kane, 2003)."

Minor corrections in the article:

Within the abstract there is an error in the following sentence: "We derive a relation between the parameters of the CRSA and MSU models for rigidities above about $10 \mathrm{GV}$ (drift effects are ignored) during periods of low to approximately average levels of solar activity." The correct sentence is: "We derive a relation between the parameters of the CRSA and MSU models for rigidities above about $10 \mathrm{GV}$ during periods of low to approximately average levels of solar activity." Also, there is an error in the expression: "i) the model parameters do not depend on rigidity". The correct expression is: "i) the model parameters do not depend on rigidity and time".

Section 4.1.2, paragraph 2: the expression "By substitution of the expression for $\ln \left(f_{\text {LIS }}(P) / f(P)\right)$ from Equation (21) in Equation (24), and taking into account Equation (2), one gets:" should be replaced by: "By substitution of the expression for $\ln \left(f_{\mathrm{LIS}}(P) / f(P)\right)$ from Equation (21) in Equation (24) we get:"

Section 5, paragraph 3: the sentences "Drift effects become negligible at $P \cong E$ and in this case $P_{0}$ and $\Delta$ are almost independent on the rigidity $P$ of the particles (Nymmik, Panasyuk, and Suslov, 1996)." should be replaced by: " $P_{0}$ and $\Delta$ are almost independent of the rigidity $P$ of the particles at $P \cong E$ (Nymmik, Panasyuk, and Suslov, 1996)."

Section 6, paragraph 3: the sentences "A relation is derived between the parameters of the CRSA and MSU models for rigidities above about $10 \mathrm{GV}$ (drift effects are ignored) during periods of low to approximately average levels of solar activity." should be replaced by: "A relation is derived between the parameters of the CRSA and MSU models for rigidities above about $10 \mathrm{GV}$ during periods of low to approximately average levels of solar activity."

Section 6, paragraph 3: the expression "i) $\alpha$ and $\beta$ are not rigidity-dependent parameters" should be replaced by: "i) $\alpha$ and $\beta$ are not time- and rigidity-dependent parameters". 